\title{
Quantum size effects in ultrathin epitaxial Mg films on $\mathrm{Si}(111)$
}

\author{
L. Aballe, C. Rogero, and K. Horn \\ Fritz-Haber-Institut der Max-Planck-Gesellschaft, Faradayweg 4-6, D-14195 Berlin, Germany
}

(Received 10 July 2001; published 12 March 2002)

\begin{abstract}
Highly perfect ultrathin epitaxial $\mathrm{Mg}(0001)$ films can be grown on $\mathrm{Si}(111) 7 \times 7$ by low-temperature deposition and annealing, as demonstrated using angle-resolved photoelectron spectroscopy and low-energy electron diffraction. This is in contrast to films grown at room temperature, which present an interfacial silicide and subsequent growth of a disordered $\mathrm{Mg}$ metallic film. The wave-vector-dependent electronic structure of the well-ordered films is investigated in detail, as a function of overlayer thickness. The spectra exhibit a number of thickness-dependent discrete peaks in the region of the magnesium $s p$ band for films up to 44 ML thick. These are caused by electron confinement within the $\mathrm{Mg}$ overlayer and are identified as quantum-well states derived from the magnesium $s p$ band. They are interpreted in terms of the phase-accumulation model which, together with the Mg band structure, is found to account for all the main features in the spectra. An estimate of the decay length of the $\operatorname{Mg}(0001)$ surface state wave function is obtained from its binding energy dependence on film thickness. Sharp intensity changes at about $20 \mathrm{ML}$ thickness point to a strain-driven thicknessdependent structural transition at this coverage.
\end{abstract}

DOI: 10.1103/PhysRevB.65.125319

PACS number(s): 73.20.At, 79.60.Dp

\section{INTRODUCTION}

Quantum well phenomena resulting from the confinement of electrons in thin metal films ${ }^{1-5}$ are not only interesting from a fundamental point of view, but can result in macroscopic effects as in the case of thickness-dependent oscillatory coupling between two ferromagnetic layers separated by a nonmagnetic spacer. ${ }^{6}$ The increasing miniaturization in the microelectronic and optoelectronic industry makes an understanding of quantum-size effects crucial for the development of future devices with a "tailored" electronic response. The mechanisms governing the growth modes of thin epitaxial films are therefore of technological as well as basic interest; the structural properties of ultrathin metals can in many ways deviate from those of the bulk materials. On the one hand, structural quantum-size effects can occur due to the reduced dimensionality, and the presence of a supporting substrate can lead to further modifications, such as interface reactions, strain, or pseudomorphism. On the other hand, in an overlayer growth process away from thermodynamical equilibrium (as necessary for growth to take place), steering of the kinetic parameters of the process can drastically influence the thin-film morphology, as will be demonstrated below.

Most studies of quantum well phenomena in thin metal films have so far concentrated on metal-on-metal systems, ${ }^{1,4,5}$ mostly involving noble metals. Thin metal films on semiconductors, on the other hand, may be the basis for novel devices utilizing quantum-well states. This paper concentrates on such a system: the widely used silicon is used as semiconducting substrate on which $\mathrm{Mg}$ films are grown. An analysis of the structure of the overlayer system $\mathrm{Mg} / \mathrm{Si}(111)$ is carried out, based on the strong influence that factors such as thickness, crystallinity, interface sharpness, or surface quality have on the electronic structure of thin metallic films. It is demonstrated that, under an appropriate choice of growth parameters, epitaxial films with an abrupt interface can be prepared also for a reactive system such as $\mathrm{Mg} / \mathrm{Si}$. This permits the observation of quantum-well states for films up to more than $40 \mathrm{ML}$ thick, and an estimation of the $\operatorname{Mg}(0001)$ surface state wave function decay length.

\section{EXPERIMENTAL DETAILS}

The measurements were carried out on the 1-m SeyaNamioka monochromator beamline at the BerlinerElektronen-Speicherring-Gesellschaft für Synchrotron Strahlung (BESSY) storage ring using a commercial angleresolving electron spectrometer (ADES400 from $V G$ ) at a base pressure of $7 \times 10^{-11}$ mbar $\left(\leqslant 9 \times 10^{-10}\right.$ mbar during $\mathrm{Mg}$ evaporation). The angle-resolved photoelectron spectra were measured using $p$-polarized light incident at $45^{\circ}$ and collected in the normal emission geometry, with an experimental resolution of $\simeq 100 \mathrm{meV}$. High-purity $(99.999 \%$ ) magnesium was evaporated from a homemade water-cooled Knudsen cell at rates of $\approx 1 \AA / \mathrm{min}$. The substrates were cut from a commercial $\mathrm{Si}(111)$ wafer, $p$-type doping $(B), 0.5$ $\Omega \mathrm{cm}$ (Virginia Semiconductors). The sample was cleaned by prolonged annealing at $600{ }^{\circ} \mathrm{C}$ followed by short flashes at $1200{ }^{\circ} \mathrm{C}$ by direct resistive heating, until it presented a sharp $7 \times 7$ low-energy electron diffraction (LEED) pattern and well-defined surface states in the photoemission spectra. Sample cooling was achieved via a liquid-nitrogen cold finger.

\section{RESULTS AND DISCUSSION}

A growth series of $\mathrm{Mg}$ on $\mathrm{Si}(111) 7 \times 7$ at room temperature is shown in Fig. 1. The spectra were recorded in normal emission for increasing $\mathrm{Mg}$ coverage in steps of $2.4 \AA$ $(\simeq 0.9 \mathrm{ML})$. Upon magnesium deposition there is an intensity increase at the Fermi energy $E_{F}$, until a Fermi edge is fully developed. Other than that, the only recognizable feature is a peak at $E_{b}=-1.7 \mathrm{eV}$, which is reminiscent of the $\operatorname{Mg}(0001)$ surface state at $\bar{\Gamma}$ (see below), but has a much broader line shape. No LEED pattern was detected for roomtemperature deposited layers. 


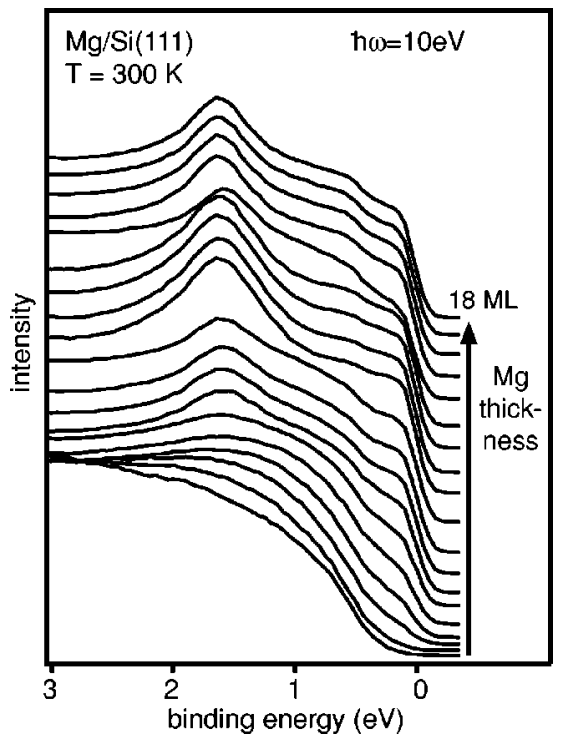

FIG. 1. Normal emission spectra of a room-temperature $\mathrm{Mg}$ / $\mathrm{Si}(111)$ deposition series. The spectra correspond to increasing coverage in intervals of $2.4 \AA$ (equivalent to $\simeq 0.9 \mathrm{ML} \mathrm{Mg}$ ).

Magnesium is known to react rather strongly with $\mathrm{Si}$ when deposited at room temperature, forming a silicide on top of the substrate. ${ }^{7-9}$ A layer of epitaxial $\mathrm{Mg}_{2} \mathrm{Si}$ is formed up to a critical thickness, and upon further deposition, growth of a disordered $\mathrm{Mg}$ layer on top of the silicide is observed. The Fermi edge in the spectra of Fig. 1 is, therefore, due to emission from the disordered metallic $\mathrm{Mg}$ layer. The silicide layer between the Mg film and the Si substrate acts as a barrier for mutual interdiffusion of $\mathrm{Si}$ and $\mathrm{Mg}$ atoms. ${ }^{9}$ However, silicide formation also prevents the growth of a crystalline metallic film.

Annealing of a $\mathrm{Mg}$ layer grown at room temperature at temperatures lower than $200{ }^{\circ} \mathrm{C}$ produces no changes in the spectra. Short annealing at $200^{\circ} \mathrm{C}$ causes a partial desorption of the layer, with the Fermi edge almost completely extinguished, but still no LEED pattern is observed. Only after further annealing to higher temperatures the metallic $\mathrm{Mg}$ layer completely desorbs and a three-domain $3 \times 1$ pattern is observed, coexisting with the $1 \times 1$ pattern from the silicon substrate. The $3 \times 1$ pattern has been found previously after annealing very thin $\mathrm{Mg} / \mathrm{Si}(111)$ films $(2-4 \AA)$ prepared at room temperature ${ }^{7}$ and upon $\mathrm{Mg}$ evaporation on $\mathrm{Si}(111) 7$ $\times 7$ at more elevated substrate temperatures $\left(>200^{\circ} \mathrm{C}\right),{ }^{8,9}$ where no silicide formation or magnesium accumulation occurs. It has been assigned by Quinn and Jona to a submonolayer-induced surface reconstruction of the silicon surface, equal to the $3 \times 1$ phases produced by adsorption of $\mathrm{Li}, \mathrm{Na}$, or $\mathrm{Ag}$ on $\mathrm{Si}(111) 7 \times 7 .{ }^{10}$ Our data confirm that it is not possible to create an ordered $\mathrm{Mg}$ film on $\mathrm{Si}(111)$ if the metal deposition has been performed at room temperature.

In order to achieve the growth of an epitaxial $\mathrm{Mg}$ overlayer, it is therefore important to reduce the reactivity and interdiffusion of $\mathrm{Mg}$ and $\mathrm{Si}$ during the initial stages of film growth. The nucleation density for island growth is strongly influenced by deposition kinetics, among other things. High rates of atom arrival on the surface and low diffusion lengths

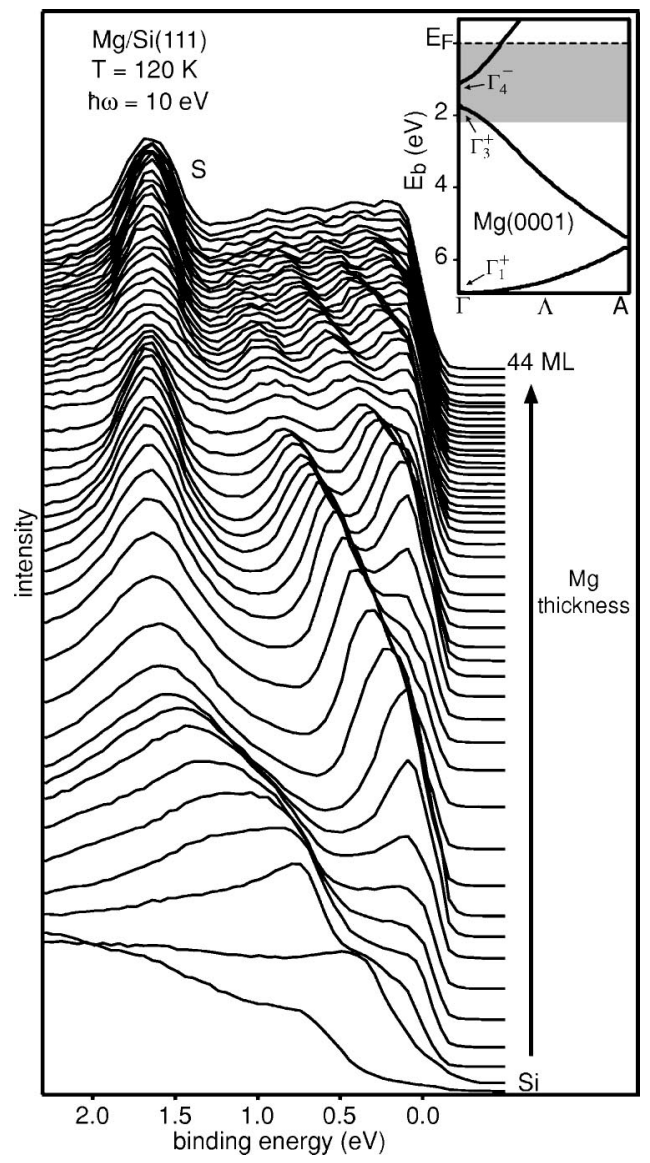

FIG. 2. Low-temperature deposition series for $\mathrm{Mg} / \mathrm{Si}(111)$, recorded in normal emission. Again the spectra correspond to increasing coverage in intervals of $\simeq 0.9 \mathrm{ML} \mathrm{Mg}$. Inset: Band structure of magnesium in the 0001 direction. The shaded region corresponds to the energy range of the photoemission spectra.

lead to higher nucleation density. Substrate temperature affects both the diffusion lengths and the balance between the different diffusion barriers that may influence growth, playing a decisive role in the growth and morphology of thin films. A higher nucleation density enhances the attempt frequency for step barrier crossing, giving rise to an enhanced interlayer mass transport and so favoring 2D growth. An attempt to inhibit interface reaction can be made by lowering the substrate temperature to a regime where nevertheless diffusion of the impinging atoms is still possible, in order to avoid poor ordering in the adsorbate layers. Lowtemperature deposition and subsequent mild annealing is a method that has been shown to work for several other metalon-semiconductor systems. ${ }^{11,12}$ Our data demonstrate that such a kinetic pathway leads to excellent epitaxial growth of Mg layers on $\mathrm{Si}(111)$.

Normal emission spectra of a growth series of epitaxial $\mathrm{Mg}$ films on $\mathrm{Si}(111) 7 \times 7$ of up to $44 \mathrm{ML}$ at a substrate temperature of $120 \mathrm{~K}$ are shown in Fig. 2. Mg deposition was done in the same steps of $\simeq 0.9 \mathrm{ML}$ as in the roomtemperature case. Striking differences between room- and low-temperature growth are clearly visible by comparing Figs. 1 and 2. The Fermi edge develops much earlier for growth at low temperature, and the appearance of the 
$\operatorname{Mg}(0001)$ surface state and of up to five quantum-well states can be followed. The surface state is the deep-lying peak marked $S$, which eventually reaches a fixed binding energy value of $1.65 \pm 0.02 \mathrm{eV}$ with respect to the Fermi energy. This is the well-known $\mathrm{Mg}(0001)$ surface state, located in the energy gap of the projected bulk band structure at the surface Brillouin zone center $\bar{\Gamma}$ first reported by Karlsson et al. ${ }^{13}$ Its development with film thickness will be discussed below. The normal emission spectra of the low-temperature growth series exhibit well-defined separate peaks in the quasifree-electron $s p$ band region, in increasing numbers with overlayer thickness.

These bulk-derived states result from electron confinement to the ultrathin film, and are well described within a simple model of a one-dimensional quantum well and the $\mathrm{Mg}$ band structure. The photoemission peaks correspond to the discrete set of the normal component of the electron wave vector $k_{\perp}$ allowed for each thickness. This can be readily understood within the phase-accumulation model: ${ }^{14,15}$ the metallic film is considered as a 1D potential well in the direction normal to the surface, where the quasifree valence electrons are confined between the vacuum and substrate. Only those $k_{\perp}$ values fulfilling the stationary state condition

$$
2 k d+\Phi_{B}+\Phi_{C}=2 \pi n
$$

are allowed. Here $n$ is an integer, the film thickness is $d$ $=N a$ ( $a$ is the interlayer distance, $N$ is the number of atomic layers) and $\Phi_{C}$ and $\Phi_{B}$ are the phase shifts at the metal/ substrate interface and metal surface, respectively. The phase shift upon reflection at the surface barrier (image potential) is well described by a semiempirical formula ${ }^{16}$ (see below). The phase shift upon reflection at the metal/substrate interface depends on the band structures of both materials and is, in general, energy as well as wave-vector dependent. ${ }^{17}$

In many cases, even the simple phase-accumulation model is sufficient to extract substantial structural information from the photoemission spectra of thin films. A diffuse interface causes nonspecular reflection of the electron waves, and thus destroys their phase coherence. Variations in the number of atomic layers lead to a superposition of emission from states derived from layers of different thicknesses, inhibiting the observation of quantum-size effects in experiments such as ours, which average over regions of $\sim 1 \mathrm{~mm}^{2}$. Therefore, an abrupt interface and a narrow height distribution are crucial for the observation of quantum-well peaks in the spectra. The width and intensity of the photoemission features, ${ }^{18}$ as well as the dispersion behavior, can in many cases be used to gauge film quality.

In the raw data of Fig. 2 the appearance and shift of the surface state and of the discrete film states can be easily followed, they shift closer to each other and increase in number as the film thickness (quantum-well width) increases, as expected from elementary quantum mechanics. The first quantum-well peak shifts gradually to higher binding energy, as expected, up to $1.13 \mathrm{eV}$. This is the highest binding energy measured for states in the upper $s p$ band branch, in agreement with theoretical calculations and the photoemission work on $\mathrm{Mg}$ single crystals of Karlsson et al., ${ }^{13}$ which locate the band edge of the upper $s p$ band branch $\left(\Gamma_{4}^{-}\right)$at $E_{b}$ $=1.1 \mathrm{eV}$. After reaching this point, the intensity of the quantum-well peak almost vanishes, so that it is hardly distinguishable above the background noise, and the deepest quantum-well peak is detected at $E_{b}=0.9 \mathrm{eV}$. From these data it is obvious that there is a finite reflectivity at the $\mathrm{Mg} / \mathrm{Si}$ interface even in the absence of an energy gap. Discrete film states are detected not only in the region of the Schottky barrier, where the substrate presents an energy gap, but also at higher binding energy. In this energy range there exist electronic states in the substrate band structure to which the quasifree conduction electrons of $\mathrm{Mg}$ can partially couple, such that the confinement is somewhat less effective. Partial confinement of electrons in the metal overlayer results in so-called quantum-well resonances, which appear broader and, in general, weaker than quantum-well states in the photoemission spectra. Here we use the generic term quantumwell states for both types of states. In the absence of an energy gap, different symmetry of the states or wave-vectordependent coupling can give rise to a finite reflectivity at the interface. Confinement behavior has been observed even for systems without any of these conditions, just due to a step in the inner potential of the overlayer and substrate and a "trapping" of the photon field in the overlayer for wavelengths corresponding to its plasmon excitations. ${ }^{19}$ This effect is also expected to play a role in the $\mathrm{Mg} / \mathrm{Si}$ system, since our data were recorded at a photon energy of $10 \mathrm{eV}$, very near the plasmon energy of $\mathrm{Mg}, \hbar \omega_{p}=10.7 \mathrm{eV}$. The exact dependence of the photoemission spectra on the photon energy and an analysis of the energy and angle-resolved photoyield of the Mg films will be presented in a forthcoming publication.

An approximate measurement of the deposition rate was obtained from a quartz thickness monitor, and the real thickness for each coverage was then calculated from the spectra, making use of our knowledge of the $\mathrm{Mg}$ band structure (see inset of Fig. 2). Since the upper branch of the $s p$ band runs through 1/4 of the Brillouin zone, a new quantum-well peak will appear in the spectra at the Fermi energy for every four lattice constants or $8 \mathrm{ML}$ of magnesium. A simple quantumwell peak counting gives thus a fast and reliable measurement of the average film thickness. The intensity at the Fermi edge shows clear oscillations as a function of coverage; each time a quantum-well peak falls in the wave-vector and energy range of the upper branch of the magnesium $s p$ band, a new peak is visible in the spectrum, and there is a maximum in the intensity at $E_{F}$. The regular oscillations are indicative of a smooth layer-by-layer growth [see Fig. 3(a)]. Since the deposition interval from start to the first maximum is the same as the following intervals between the maxima, the existence of an extended reacted layer between the substrate and overlayer can be ruled out. The LEED patterns of asdeposited Mg films of $\simeq 15$ ML and 44 ML are shown in Figs. 3(b) and 3(c), respectively. Both patterns are hexagonal and of high quality. The slight rotational disorder, which causes a somewhat elongated shape of the spots for the thinner film, seems to disappear upon further deposition, as the rounding of spots for the thicker film indicates.

A direct comparison of the experimental data (in a twodimensional representation of the intensity) with the pre- 


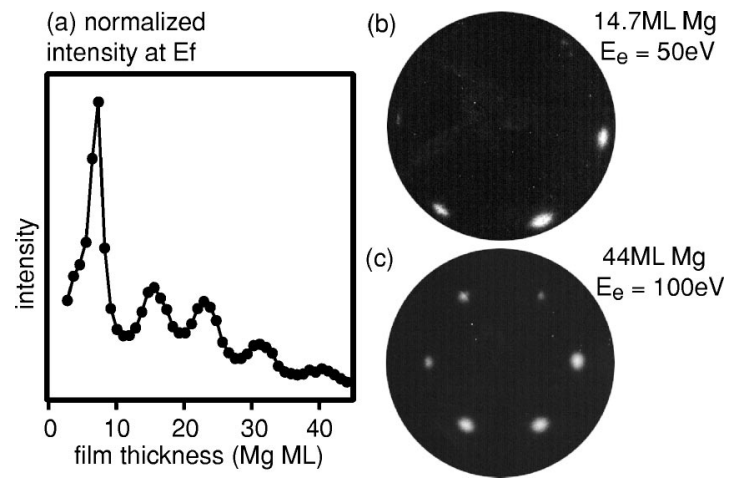

FIG. 3. (a) Intensity at the Fermi level for the low-temperature $\mathrm{Mg} / \mathrm{Si}(111)$ deposition series of Fig. 2. The photoemission intensity is normalized by dividing in each case by the photocurrent induced by the synchrotron light at the exit mirror in the beamline. (b), (c) LEED pattern at two different stages of the deposition process.

dicted quantum-well energy positions is made in Fig. 4. The theoretical positions have been calculated with the Mg bulk band structure, ${ }^{20}$ within the phase-accumulation model [Eq. (1)]. The phase shift at the surface barrier (image potential) $\Phi_{B}$ has been computed with the semiempirical formula ${ }^{16}$

$$
\Phi_{B}=\pi \sqrt{\frac{3.4 \mathrm{eV}}{E_{V}-E}}-\pi,
$$

where $E_{V}$ is the vacuum level. This model already gives very good agreement through most of the energy range studied. Small deviations from the predicted behavior are due to the contribution from the phase shift at the metal/semiconductor interface, which has not been included in this first approximation. In particular, a deviation in quantum-well states 1 and 2 for binding energies higher than $\simeq 0.7 \mathrm{eV}$ could be correlated with a strong change in reflectivity associated with the end of the Schottky barrier and, therefore, the appearance of the valence-band maximum and states to which some coupling is possible. Actually, not only the valence band maximum but any substrate band edge has an influence on the phase shift at the interface. ${ }^{17}$ The very limited energy range in which quantum-well states from the $\mathrm{Mg}$ overlayer coexist

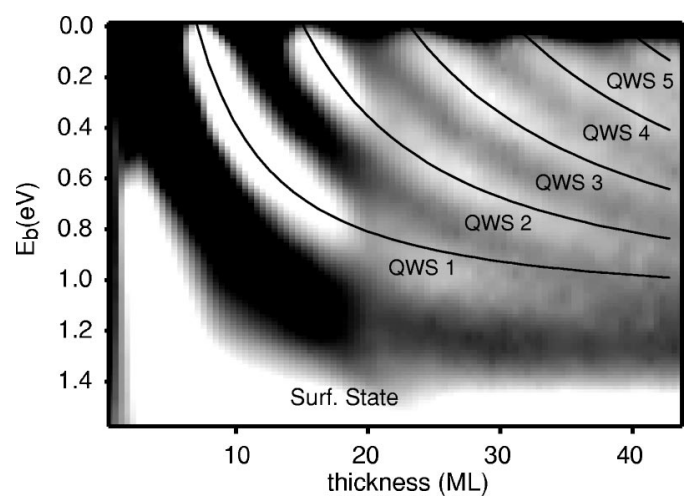

FIG. 4. (a) Intensity plot of the low-temperature $\mathrm{Mg} / \mathrm{Si}(111)$ deposition series of Fig. 2 and theoretical $k_{n}$ evolution with thickness (see text). The spectra are scaled to a common average intensity. with substrate bands prevents a systematic analysis of such an effect for the $\mathrm{Mg} / \mathrm{Si}(111)$ system.

We can conclude that low-temperature $\mathrm{Mg}$ deposition on $\operatorname{Si}(111) 7 \times 7$ favors the growth of epitaxial, twodimensional, metallic $\mathrm{Mg}$ overlayers. The photoemission spectra of the overlayers are characterized by a set of discrete quantum-well peaks, derived from the $\mathrm{Mg} s p$ band due to electron confinement. The quantized states shift to higher binding energy and closer to each other with increasing thickness; a new state crosses the Fermi edge for every eight $\mathrm{Mg}$ monolayers. The presence of clear, regular, and narrow quantum-well peaks already in the spectra of the asdeposited layers indicates that monocrystalline films of great homogeneity are created, and that, by reducing the substrate temperature, the interface reaction is inhibited or at least strongly reduced. For the thickest films, the quantum-well states are less than $300 \mathrm{meV}$ apart, and have widths of $\simeq 100 \mathrm{meV}$ only. This is at the limit of our experimental resolution and is in the range of the width measured for quantum-well states in atomically uniform $\mathrm{Ag}$ films. ${ }^{4}$ It is remarkable that unannealed films of more than 40 atomic layers still have such a crystalline quality and low roughness such that quantum-well peaks can be distinguished, in contrast to room-temperature growth, which leads to a disordered layer of metallic $\mathrm{Mg}$.

Not only the "bulk" electronic states of Mg are affected by the reduced film thickness, but also the $\operatorname{Mg}(0001)$ surface state. Its wave function is not localized solely in the surface layer, but decays into the bulk, such that it is perturbed for thicknesses below a certain decay length, and its energy is shifted with respect to its position for bulk samples. The gap in which the surface state exists, at the $\Gamma$ point (see inset of Fig. 2), has a width of $\simeq 0.57 \mathrm{eV}$ (Ref. 20) where two freeelectron initial-state bands are split. The degree of localization is characterized by a decay length $\lambda_{i}$ sensitively related to the boundary condition at the solid-vacuum interface and the energy splitting of the surface state from the bulk continuum. We can reduce the problem to one dimension and describe the surface state by a nearly free-electron wave function, which is physically reasonable and yet analytical. ${ }^{21}$ The wave function of a surface state localized near $z=0$ for a semi-infinite solid extending over the half-space $z \leqslant 0$ then has the form

$$
\Psi_{S}(z)= \begin{cases}N e^{z / \lambda_{i}} \cos \left(k_{B Z \text { bound }} z+\delta\right), & z<0 \\ N e^{-z / \lambda_{o}} \cos (\delta), & z>0,\end{cases}
$$

where $k_{\text {BZbound }}$ is the wave-vector at the volume Brillouinzone boundary ( $\Gamma$ point in our case), $\mathrm{V}_{1}=-\mathrm{V}_{0001}>0$ and $\mathrm{V}_{0}=-\mathrm{V}_{000}>0$ with $\mathrm{V}_{000}=-6.9 \mathrm{eV}$, the inner potential. $N$ is the normalization constant, $\delta$ is the phase shift required for matching at the interface, and $\lambda_{o}$ is the decay length outside the surface. The different parameters are defined as

$$
\delta=\cos ^{-1}\left[\frac{k_{\text {BZbound }}}{\sqrt{\frac{2 m}{\hbar^{2}}\left(V_{0}+V_{1}\right)}}\right], \quad 0 \leqslant \delta \leqslant \pi / 2 \text {, }
$$




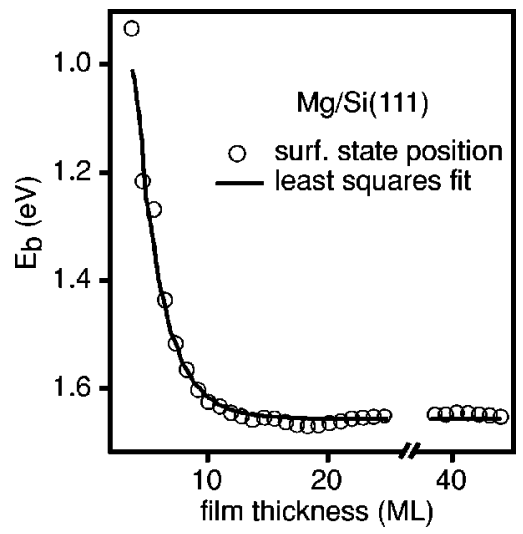

FIG. 5. Surface state energy position for low-temperature $\mathrm{Mg}$ / $\mathrm{Si}(111)$ deposition and exponential fit to the data.

$$
\begin{gathered}
1 / \lambda_{i}=\frac{V_{1}}{V_{0}+V_{1}} \sqrt{\frac{2 m}{\hbar^{2}}\left(V_{0}+V_{1}\right)-k_{\text {BZbound }}^{2},} \\
\lambda_{o}=\left(V_{1} / V_{0}\right) \lambda_{i} .
\end{gathered}
$$

The function has the correct limiting behavior in that it decays exponentially into the vacuum and oscillates into the bulk, damped more strongly for a larger gap where the surface state exists. Surface resonances, hence, have, in principle, an infinite decay length.

In Fig. 5 the energy position of the $\operatorname{Mg}(0001)$ surface state at $\bar{\Gamma}$ is plotted versus layer thickness, in order to highlight the upward shift in energy for layers thinner than the decay length of the state. We treat this effect as a perturbation in the crystal potential, caused by the presence of the substrate, consisting in a potential step in the region $\mathrm{z}<-d$, where $d$ is the $\mathrm{Mg}$ overlayer thickness. This is, of course, not the only effect of the substrate and a detailed calculation should take into account the difference in the in-plane atomic structure for both lattices as well as surface relaxation. In first-order perturbation theory, the shift in the surface state binding energy is given by $\Delta E(d)=\left\langle\Psi_{S}|\Delta V| \Psi_{S}\right\rangle$. A fit to the experimental energy positions with the resulting function is shown in Fig. 5 together with the measured peak positions. The results of the fit are $E_{b}(\infty)=1.65 \pm 0.02 \mathrm{eV}$ for the binding energy and a decay length of $\lambda_{i}=11.6 \pm 0.4 \AA$, or 4.5 $\pm 0.2 \mathrm{Mg}$ interlayer spacings. The errors correspond to the least-squares fit for the decay length, and to the typical error in the binding energy determination for $E_{B}(\infty)$. The only fit parameters are $E(\infty), \lambda_{i}$, and a multiplicative constant. The resulting decay length is very similar to that obtained for the $\mathrm{Au}(111)$ surface state with a similar procedure. ${ }^{22}$ This value should be taken only as an estimate, since the thinner $\mathrm{Mg}$ / $\mathrm{Si}(111)$ layers very likely present structural modifications (see discussion below), which could also contribute to the surface-state shift. From the decay length, the energy splitting of the surface state from the lower band gap edge (the $\Gamma_{3}^{+}$point) can be obtained by means of ${ }^{23}$

$$
\delta E=\frac{\hbar^{2} \lambda_{i}^{-2}}{2 m^{*}},
$$

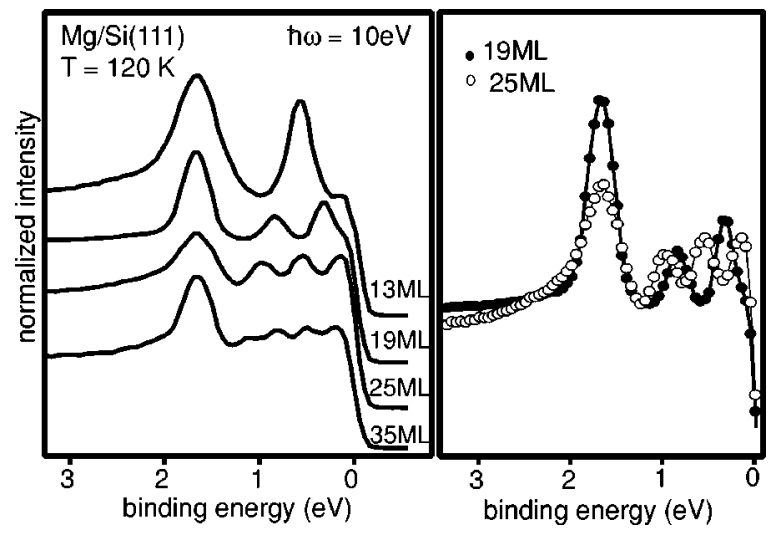

FIG. 6. Normal emission spectra of as-deposited $\mathrm{Mg} / \mathrm{Si}(111) 7$ $\times 7$ films of $13,19,25$, and $35 \mathrm{ML}$, representative of the different regimes observed (see text).

where $m^{*}$ is the effective mass associated with the lower bulk band. An effective mass of about $0.1 m_{e}$, as obtained from the calculated band structure of Ref. 20, together with the experimental $\lambda_{i}$ value, gives $\delta E=280 \mathrm{meV}$, in good agreement with a position of the band edge at $1.7 \mathrm{eV}$ binding energy. ${ }^{13,24}$ The shift of the $\mathrm{Mg} \bar{\Gamma}$ surface state towards $E_{F}$ for the thinnest layers, and its evolution towards a saturation value for thicker films, can be followed visually in the spectra of Fig. 2.

There is a striking feature in the Mg surface state evolution with film thickness. The relative intensity of surface state to quantum-well peaks, which initially monotonically increases, goes successively through a maximum (at $\sim 19 \mathrm{ML}$ ) and a minimum (at $\sim 25 \mathrm{ML}$ ). In fact, at $\sim 25 \mathrm{ML}$, the surface-state intensity passes a minimum also in absolute terms. The corresponding spectra are compared on the right-hand side of Fig. 6. On the left-hand side, spectra from $13 \mathrm{ML}$ and $35 \mathrm{ML}$ films are also shown, representative of the $\mathrm{Mg}$ films below and above the critical coverage region. The line shape of the surface state peak also undergoes considerable changes in this coverage region; it is narrower and highly symmetric at the relative intensity maximum (19 ML) and highly asymmetric at the minimum (25 ML). The asymmetry is given by a high binding energy shoulder due to, at least in part, emission from the lower branch of the $s p$ band, and therefore also of "bulk" origin, as are the quantum-well states. Beyond this thickness, the width and intensity of the surface state peak increase again. A small peak appears at $E_{b} \simeq 4.1 \mathrm{eV}$, which persists up to the thickest overlayers investigated. These sharp changes could be due to a structural transition. One possibility is that the first few magnesium monolayers grow in an fcc-like manner, and that after a critical thickness the more stable hcp configuration sets in, or, even more likely, that the first few layers grow in an (in-plane) coincidence lattice with the silicon substrate, but after a critical thickness, a strain-driven structural transition to a relaxed $\mathrm{Mg}$ lattice with ideal bulk spacing takes place. A lattice relaxation in such a narrow thickness range is likely to imply a surface with a significantly higher dislocation density than the surfaces of layers with higher and lower thickness, thus causing a "temporary" 


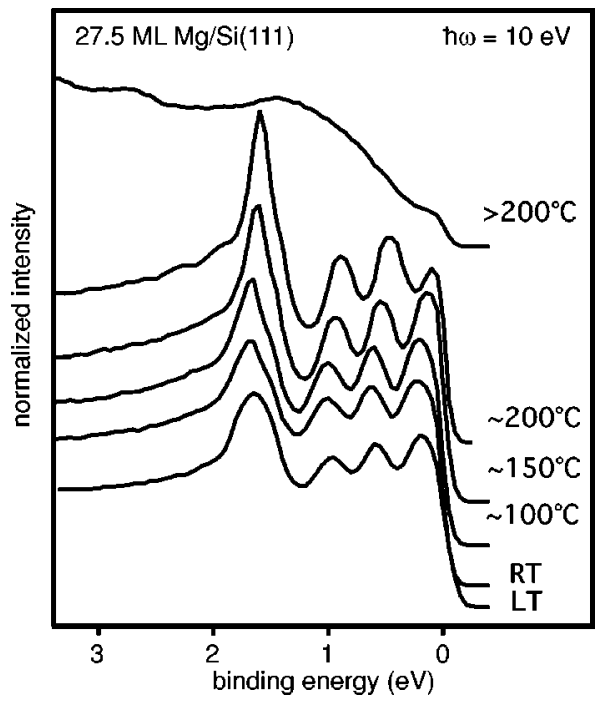

FIG. 7. Normal emission spectra from a 27.5-ML $\mathrm{Mg} / \mathrm{Si}(111) 7 \times 7 \mathrm{film}$. The film was deposited at $120 \mathrm{~K}$ and annealed for 3-5 minutes at increasing temperatures up to the breaking up into islands and ultimately complete magnesium desorption.

surface state depletion. In the $\mathrm{Mg} / \mathrm{Si}(111)$ system, the deviation from a 5:6 relation in the in-plane lattice constants [(111) for $\mathrm{Si}$ and (0001) for $\mathrm{Mg}$ ] is only $0.4 \%$, so that a coincidence lattice could be reached with only a slight $\mathrm{Mg}$ lattice contraction. A contraction of the $\mathrm{Mg}$ lattice during the first few monolayers has also been observed in the growth of epitaxial magnesium islands on $\mathrm{H}$-terminated $\mathrm{Si}(111) .{ }^{25} \mathrm{~A}$ modified lattice during the initial growth stages might be substrate stabilized. This would involve a substrate-metal interaction strong enough to force a $\mathrm{Mg}$ lattice structure different from its equilibrium one. The LEED pattern for a $44 \mathrm{ML}$ $\mathrm{Mg} / \mathrm{Si}$ (111) film [see Fig. 3(c)] shows a clear sixfold symmetry, while for thinner films we cannot clearly distinguish between a sixfold and a threefold symmetry pattern. More detailed structural and electronic structure investigations are necessary to confirm the existence of a structural transition, and to elucidate its nature.

Once a magnesium overlayer has been deposited at reduced temperature, it remains stable upon annealing to room temperature and above, in contrast to the films grown directly at room temperature. Spectra corresponding to the asdeposited overlayer as well as at several stages of the annealing series of a $27.5 \mathrm{ML}$ thick film are shown in Fig. 7. At first, short anneals to temperatures below $100^{\circ} \mathrm{C}$ do not cause any major change of the electronic features. Above $\sim 100^{\circ} \mathrm{C}$, several changes take place. The surface state peak gains intensity, its line shape becomes highly asymmetric and shifts slightly from $-1.67 \mathrm{eV}$ at low temperature to $-1.61 \mathrm{eV}$ just before desorption. The quantum-well peaks become somewhat more intense and shift towards the Fermi energy, such that they correspond to a slightly lower thickness in the phase-accumulation picture.

Finally, in the spectra of the annealed layer, emission from a couple of quantum-well states at higher binding energy than the surface state, derived from the lower branch of the $s p$ band, is also visible. The fact that these quantum-well

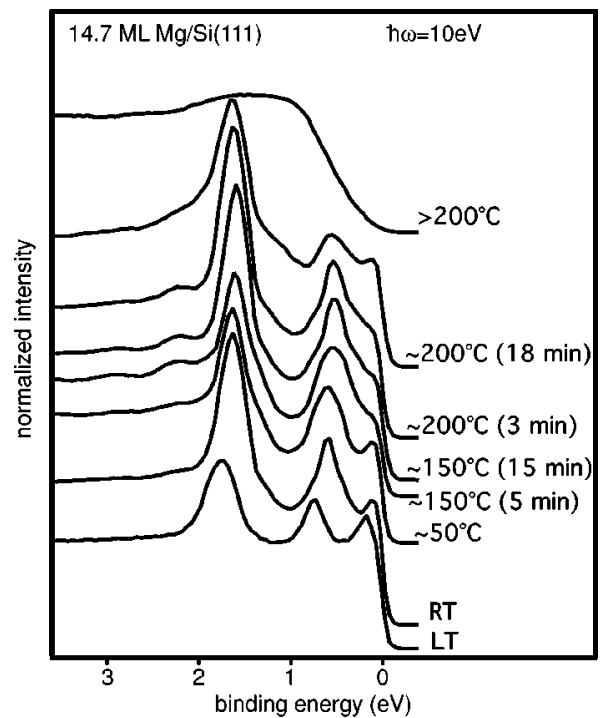

FIG. 8. Normal emission spectra of a 14.7-ML Mg/Si(111)7 $\times 7$ film. The film was deposited at $120 \mathrm{~K}$ and annealed first to room temperature within $2 \mathrm{~h}$ and then to increasing temperatures for a few minutes.

states are detected only after annealing is likely to be due to an improved crystallinity in the $\mathrm{Mg}$ layer, or it could be related to changes in the interface (electronic) structure. It is in fact likely that the structure of the $\mathrm{Mg} / \mathrm{Si}$ interface changes upon annealing, giving rise to different boundary conditions for the reflection of electron waves, and therefore modifying the condition for the existence of quantum-well states. Modified reflection properties at the interface can cause not only shifts of the quantum-well peaks, but also line shape and background shape modifications. ${ }^{17}$ The formation of a silicide at the interface would certainly modify its electronic structure and could give rise to a more effective confinement of electron waves derived from the lower $s p$ branch, as well as to the energy shift of the quantum-well peaks in the upper branch. Annealing to $\sim 200^{\circ} \mathrm{C}$ results in island formation and the consequent overall shift of the spectrum by $170 \mathrm{meV}$ to higher kinetic energies due to surface photovoltage ${ }^{26}$ since the metal is not "grounded" any more by a conductive path, having a semiconductor as substrate. The sample was kept at low temperature during measurements, so that the electronhole recombination rate is low and surface photovoltage is readily observed. A $(1 \times 1)$ hexagonal LEED pattern was observed from deposition up to the formation of islands, and after desorption of the layer, three domains of a $(3 \times 1)$ reconstruction were visible in the diffraction pattern, just as that found after desorption of a film grown at room temperature (see above). After the onset of magnesium desorption, the intensity at the Fermi edge vanishes, the spectrum, in general, being very similar to that after desorption of a thick film deposited at room temperature.

The spectra of a 14.7 ML Mg film during annealing, visible in Fig. 8, show qualitatively the same behavior: Slight line shape changes and energy shifts for moderate annealing temperatures, followed by island formation and the consequent overall shift of the spectrum to higher kinetic energies due to surface photovoltage, and finally desorption of the 
metallic layer. In this case, already at room temperature there are shifts of the quantum-well peaks towards $E_{F}$, and a pronounced asymmetry of the surface state line shape can be detected with the naked eye already after annealing to $50{ }^{\circ} \mathrm{C}$. The increasing asymmetry of the surface state goes along with the appearance of quantum-well states at its high binding energy side, just as in the previous case. The quantumwell peaks below the surface state are very pronounced in the spectra of Fig. 8 corresponding to annealing temperatures of $150^{\circ} \mathrm{C}$. The surface state position of the as-deposited $\mathrm{Mg}$ layer is $1.76 \mathrm{eV}$, at $\simeq 100 \mathrm{meV}$ higher binding energy than the "equilibrium" position of $1.65 \mathrm{eV}$, which is reached for thicker films. After annealing to room temperature within 2 $\mathrm{h}$, the equilibrium position is reached.

The shift of the surface state upon annealing could be strain related. Calculations ${ }^{11}$ have shown that small lattice variations (in the range of $1 \%$ ) lead to shifts in the bulk band edges that can cause surface state energy shifts in the range of $100 \mathrm{meV}$. Such a shift would favor the picture of a coincidence lattice with the substrate for the thinner films at low temperature, which can be lifted by annealing to room temperature, or which is relaxed also at low temperature after a critical thickness is reached (see above).

Upon further annealing, at the onset of island formation, a further shift to lower binding energy $(1.60 \mathrm{eV})$ takes place, just as for the 27.5 ML thick film. Clustering into islands involves a major mass rearrangement, so that it cannot be ruled out that the second shift is also caused by strain. Also the formation of small islands would lead to energy shifts in the surface state due to lateral localization of its wave function. ${ }^{27-29}$ After annealing to $200^{\circ} \mathrm{C}$ for a few minutes, surface photovoltage induces a $200-\mathrm{meV}$ shift of the Fermi edge. This is again indicative of island formation. Also extra spots appear in the LEED pattern at this stage, and strong line shape changes in the background of the spectra occur. The surface state and quantum-well peaks are still visible, but seem to be superimposed on a strong background. The background has the shape of the spectrum corresponding to the situation after desorption of the metal (upper most spectrum), which could correspond to a combination of an interface silicide with parts of the $3 \times 1$ reconstruction.

In general, we find that thinner films break up at lower annealing temperatures. The thinnest films studied (3 ML $\mathrm{Mg}$ ) break into islands already below room temperature, while a 44 ML layer remains stable upon annealing to $\sim 200^{\circ} \mathrm{C}$. There is another important piece of information that can be extracted from Fig. 8: the influence of the time factor. Comparing the spectrum of the $\mathrm{Mg}$ film annealed for $5 \mathrm{~min}$ to $150{ }^{\circ} \mathrm{C}$ with that annealed for $15 \mathrm{~min}$ (or those annealed for $3 \mathrm{~min}$ or $18 \mathrm{~min}$ to $200^{\circ} \mathrm{C}$ ), it can be concluded that once a certain temperature is reached, changes continue taking place on a time scale of (at least) minutes. Recently, a novel thin-film growth mechanism has been proposed, ${ }^{30}$ in which the electronic contribution to the total film energy is responsible for the existence of "magical" or critical heights, and some experimental evidence has been gathered in this sense. ${ }^{31-33}$ Within our experimental resolution, we have not found evidence of the existence of preferred film thicknesses in the system $\mathrm{Mg} / \mathrm{Si}(111)$. Keeping in mind that the ability of photoemission for morphology analysis is limited, we have to conclude from our data that in the growth of $\mathrm{Mg}$ on $\mathrm{Si}$, kinetic and interface effects are dominating over the more subtle electronic contribution to film energy.

Mild annealing of the $\mathrm{Mg}$ films grown at low temperature thus slightly increases their quality and allows for relaxation of the strain present in the thinner layers. For each overlayer thickness there is a critical temperature (increasing with increasing film height) above which clustering and eventually desorption take place. Since the critical temperature is above room temperature for any thickness above a couple of monolayers, epitaxial $\mathrm{Mg} / \mathrm{Si}(111)$ films stable at room temperature can be obtained for a wide range of thicknesses.

\section{SUMMARY}

We have shown that the growth of two-dimensional, monocrystalline films of $\mathrm{Mg}$ with (0001) orientation on $\mathrm{Si}(111)$ with an abrupt and homogeneous interface is possible by depositing the metal at low temperature. The shift of the $\operatorname{Mg}(0001)$ surface state for the thinnest layers permits an estimation of its decay length. The occurrence of quantumwell states for a wide range of thicknesses and their appropriate description by a simple phase-accumulation model permit the investigation of the structural properties of the films, their evolution with film thickness, and the changes upon annealing.

\section{ACKNOWLEDGMENTS}

We thank C. H. Chen, H. Haak, and the BESSY staff for technical support. This work has been supported by the Bundesministerium für Bildung und Forschung under Grant No. 05SE8OLA7.
${ }^{1}$ S. Lindgren and L. Walldén, Phys. Rev. Lett. 59, 3003 (1987).

${ }^{2}$ M. Jałochowski, H. Knoppe, G. Lilienkamp, and E. Bauer, Phys. Rev. B 46, 4693 (1992).

${ }^{3}$ D. Evans, M. Alonso, R. Cimino, and K. Horn, Phys. Rev. Lett. 70, 3483 (1993).

${ }^{4}$ J. Paggel, T. Miller, and T. Chiang, Science 283, 1709 (1999).

${ }^{5}$ R. Kawakami, E. Rotenberg, H. Choi, E. Escorcia-Aparicio, M. Bowen, J. Wolfe, E. Arenholz, Z. Zhang, N. Smith, and Z. Qiu, Nature (London) 398, 132 (1999).
${ }^{6}$ J. Ortega and F. Himpsel, Phys. Rev. Lett. 69, 844 (1992).

${ }^{7}$ J. Quinn and F. Jona, Surf. Sci. 249, L307 (1991).

${ }^{8}$ C. Wigren, J. Andersen, R. Nyholm, and U. Karlsson, Surf. Sci. 289, 290 (1993).

${ }^{9}$ K. An, R. Park, J. Kim, C. Park, S. Lee, T. Abukawa, S. Kono, T. Kinoshita, A. Kakikazi, and T. Ishii, J. Appl. Phys. 78, 1151 (1995).

${ }^{10}$ W. Fan and A. Ignatiev, Phys. Rev. B 41, 3592 (1990).

${ }^{11}$ G. Neuhold and K. Horn, Phys. Rev. Lett. 78, 1327 (1997). 
${ }^{12}$ L. Aballe, C. Rogero, S. Gokhale, S. Kulkarni, and K. Horn, Surf. Sci. 482-485, 488 (2001).

${ }^{13}$ U. Karlsson, G. Hansson, P. Persson, and S. Flodström, Phys. Rev. B 26, 1852 (1982).

${ }^{14}$ P. M. Echenique and J. B. Pendry, J. Phys. C 11, 2065 (1978).

${ }^{15}$ N. Smith, Phys. Rev. B 32, 3549 (1985).

${ }^{16}$ E. McRae and M. Kane, Surf. Sci. 108, 435 (1981).

${ }^{17}$ L. Aballe, C. Rogero, P. Kratzer, S. Gokhale, and K. Horn, Phys. Rev. Lett. 87, 156801 (2001).

${ }^{18}$ K. Meinel, A. Beckmann, M. Klaua, and H. Bethge, Phys. Status Solidi A 150, 521 (1995).

${ }^{19}$ S. Barman, P. Häberle, K. Horn, J. Maytorena, and A. Liebsch, Phys. Rev. Lett. 86, 5108 (2001).

${ }^{20}$ L. Wilk, W. Fehler, and S. Vosko, Can. J. Phys. 56, 266 (1978).

${ }^{21}$ E. Goodwin, Proc. Cambridge Philos. Soc. 35, 205 (1939).

${ }^{22}$ T. Hsieh and T. Chiang, Surf. Sci. 166, 554 (1986).

${ }^{23}$ T. Hsieh, T. Miller, and T. Chiang, Phys. Rev. Lett. 55, 2483 (1985).
${ }^{24}$ R. Bartynski, R. Gaylord, T. Gustafsson, and E. Plummer, Phys. Rev. B 33, 3644 (1986).

${ }^{25}$ K. Saiki, K. Nishita, Y. Ariga, and A. Koma, J. Vac. Sci. Technol. A 17, 2911 (1999).

${ }^{26}$ J. Demuth, W. Thompson, N. DiNardo, and R. Imbihl, Phys. Rev. Lett. 56, 1408 (1986).

${ }^{27}$ R. Fischer, T. Fauster, and W. Steinmann, Phys. Rev. B 48, 15496 (1993).

${ }^{28}$ J. Li, W. Schneider, R. Berndt, and S. Crampin, Phys. Rev. Lett. 80, 3332 (1998).

${ }^{29}$ M. Giesen, G. S. Icking-Konert, and H. Ibach, Phys. Rev. Lett. 82, 3101 (1999).

${ }^{30}$ Z. Zhang, Q. Niu, and C. Shih, Phys. Rev. Lett. 80, 5381 (1998).

${ }^{31}$ A. Smith, K. Chao, Q. Niu, and C. Shih, Science 273, 226 (1996).

${ }^{32}$ V. Yeh, L. Berbil-Bautista, C. Wang, K. Ho, and M. Tringides, Phys. Rev. Lett. 85, 5158 (2000).

${ }^{33}$ D. Luh, T. Miller, J. Paggel, M. Chou, and T. Chiang, Science 292, 1131 (2001). 